\title{
Field Assessment of a Model for Fungicide Effects on Intraplant Spread of Stem Rust in Perennial Ryegrass Seed Crops
}

\author{
W. F. Pfender and J. Eynard
}

U.S. Department of Agriculture-Agricultural Research Service, National Forage Seed Production Research Center and Oregon State University Department of Botany and Plant Pathology, 3450 SW Campus Way, Corvallis 97331. Accepted for publication 23 January 2009.

\section{ABSTRACT}

Pfender, W. F., and Eynard, J. 2009. Field assessment of a model for fungicide effects on intraplant spread of stem rust in perennial ryegrass seed crops. Phytopathology 99:696-703.

Intraplant spread of stem rust (Puccinia graminis subsp. graminicola) in perennial ryegrass during tiller extension is a major determinant of epidemic severity and is dominated by stem extension dynamics. Simple equations for extension of inflorescence and internodes are presented and parameterized. These equations are combined with previously published equations for pathogen latent period and for postinfection efficacy of fungicides to produce a model for effects of fungicide type and timing on intraplant spread. The model is driven by thermal units, calculated from air temperature measurements. Three field experiments, conducted independently from the field experiments that provided data for plant growth model parameterization, were conducted to assess performance of the disease spread model. Either propiconazole or azoxystrobin, the two most commonly used fungicides for stem rust control, was applied to tillers that had stem rust pustules on the flag sheath and in which the inflorescence was partially extended. Intraplant spread of disease to the extending inflorescence (stem and flowerhead) was observed at several dates following treatment and compared with modeled severities. The model estimated accurately the severities of inflorescence infection for most treatments and observation times, with a correlation coefficient of 0.93 for modeled versus observed disease severities across the three experiments. The model correctly estimated the rank order of final severities among the treatments (fungicide type and timing). The model can be extended to intraplant spread of stem rust at all internodes and incorporated into decision support tools for fungicide type and timing in management of this disease.

Additional keywords: disease management.
Models of disease development that incorporate the effects of fungicide treatment can be useful in developing strategies and in implementing tactics for optimum management of plant diseases. Such models have not yet been developed for foliar diseases of grasses grown for seed. One of these diseases, stem rust caused by Puccinia graminis subsp. graminicola, is the major disease constraint to production of grass seed in the northwestern United States, where most of North America's perennial ryegrass seed (Lolium perenne) is harvested (12). The rust pathosystem in grass seed crops is similar in many respects to rust diseases in wheat. A model of fungicide effects on wheat yield loss from rusts and other foliar disease (4) includes a component that specifies activity of fungicides as a function of dose and timing, as well as components for host growth and relationship of photosynthetic area to yield.

A unique feature of stem rust disease in grasses, and possibly in cereal grains, is the manner in which spread of the pathogen on the plant is facilitated by the geometry and mechanics of plant growth during tiller extension (8). Each leaf of a grass plant has a blade and a sheath, the latter forming a hollow cylinder enclosing successively younger leaves, stem, and inflorescence (Fig. 1). During tiller extension, each segment emerges from within the next-oldest internode, similar to a telescope being opened. If $P$. graminis is present as a sporulating pustule on the outer surface of a leaf sheath, it can release urediniospores from the inner surface of the sheath cylinder also, depositing spores on the next-younger

Corresponding author: W. F. Pfender; E-mail address: pfenderw@onid.orst.edu

doi:10.1094/PHYTO-99-6-0696

This article is in the public domain and not copyrightable. It may be freely reprinted with customary crediting of the source. The American Phytopathological Society, 2009. elongating internode that is emerging from within the infected sheath (8). In this way, a single stem rust lesion on a leaf sheath can infect at multiple, contiguous sites on the enclosed stem or inflorescence as it elongates (Fig. 1). After a latent period has passed, the resultant elongated, extensive areas of rust pustules on the stem and inflorescence can account for $>50 \%$ of disease severity in an affected perennial ryegrass seed field (9). Effective epidemic modeling and management of stem rust with fungicides requires a quantitative understanding of this intraplant spread process and the manner in which it is affected by fungicides. We have previously characterized the activity and physical modes of action of the two fungicide classes most commonly used for managing stem rust in grasses grown for seed (9). The efficacy of propiconazole and azoxystrobin fungicides, considered in both pre-infection and postinfection applications, was described as a function of the time duration between infection and application. We also noted that, when azoxystrobin is applied to a stem rust lesion on a leaf sheath, there is a high probability of eliminating further intraplant spread because it inhibits sporulation from the inner-sheath surface of the pustule; when propiconazole is applied, this effect occurs with a much lower probability (9). Because of this difference, and because the fungicides differ also in postinfection activity, overall fungicide efficacy during tiller extension will depend critically on choice of fungicide and the timing of its application in relation to the timing of infections and internode elongation.

We are constructing an epidemic model for stem rust, and components of it have been previously published $(5,6,8)$. The model will require an integration of fungicide effects (9) with plant growth dynamics. Some growth models for other members of Gramineae such as wheat include consideration of the elongation of internodes, whether as stem sections (10) or as distances 
between successive leaf layers (2). Here, we present a simple model for stem elongation in perennial ryegrass. The stem elongation equations were combined with previously published equations for fungicide effects (9) and latent period duration (5) to model effects of fungicide application on development of stem rust disease in extending tillers. The utility of the combined model was assessed in field experiments where we measured the severity of disease due to intraplant spread from infected sheath to extending inflorescence, with and without the influence of fungicide sprays.

\section{MATERIALS AND METHODS}

Stem elongation. Data describing the dynamics of tiller extension were obtained by frequent, repeated observations on individual tillers growing in the field. Each tiller was chosen at random from within a stand of perennial ryegrass cv. Morningstar that was planted in autumn and maintained according to standard agronomic practices (7), including adequate $\mathrm{N}$ fertilizer. Each selected tiller was marked in late winter by tying a small thread around the third-highest (i.e., third-youngest) leaf. At approximately weekly intervals starting in March, the length of each successively emerging plant segment (leaf sheath, exposed stem, or inflorescence) (Fig. 1) was recorded. The thread was relocated to progressively newer leaves as the older leaves died. Individual records of number and lengths of plant segments on each date were kept for each tiller. At the end of the season, the data set for each tiller was used to reconstruct the growth dynamics for the plant segments, numbered from sheath 1 (the latest, or flag, leaf sheath) to sheath 3 and from stem 0 (the inflorescence) through stem 3 (the fourth-youngest stem internode) (Fig. 1). Stems 1 to 3 were each defined as the segment between the ligules (bladesheath junction) of two successive leaves. Data were obtained in 2004, 2005, and 2007 from plantings sown the previous autumn. Ten individual tillers were followed throughout the season in 2004 and six tillers in each of the years 2005 and 2007. Air temperature at the top of the plant canopy in each planting was measured and recorded at 15-min intervals with a thermistor temperature probe (Campbell Scientific Inc., Logan UT) enclosed in a ventilated shield, as described previously (6).

Data from the three observation sets (2004, 2005, and 2007) were combined to produce one data set for each of the plant segments, and parameters were fit to each of these data sets. A sigmoidal equation, which is a common shape for many biological growth processes, was used to model the relationship of length $(L)$ versus time $(x)$ for each of the tiller segments. The equation is

$$
L=a /(1+\exp [(b-x) / c])
$$

where $a$ is the upper limit (maximum length), $b$ is the location of center of the S-shaped curve along the $x$ (time) axis, and $c$ controls the steepness of the curve. By reference to Figure 1, it can be seen that each segment of the grass plant attains some length before it becomes visible above the sheath that encloses its early growth. We modified the sigmoidal equation to model that part of the plant segment that is visible (i.e., has emerged above the next-lower plant segment). The modification was accomplished by subtracting a proportion of the value of parameter $a$, thereby truncating the curve:

$$
L=\{a /(1+\exp [(b-x) / c])\}-(d) a
$$

The coefficient for truncation $(d)$ was chosen based on our measurements of plant morphology. For example, we observed that stem 1 first appears above stem 2 when the latter is 0.18 of the length that the emerging stem 1 segment will eventually attain. Therefore, we set $d$ to 0.18 for stem 1 , modeling the situation in which 0.18 of stem 1 growth has occurred before it becomes visible and, thus, measurable. Negative values of $L$, which occur when $x$ (time) is earlier than the time at which the segment

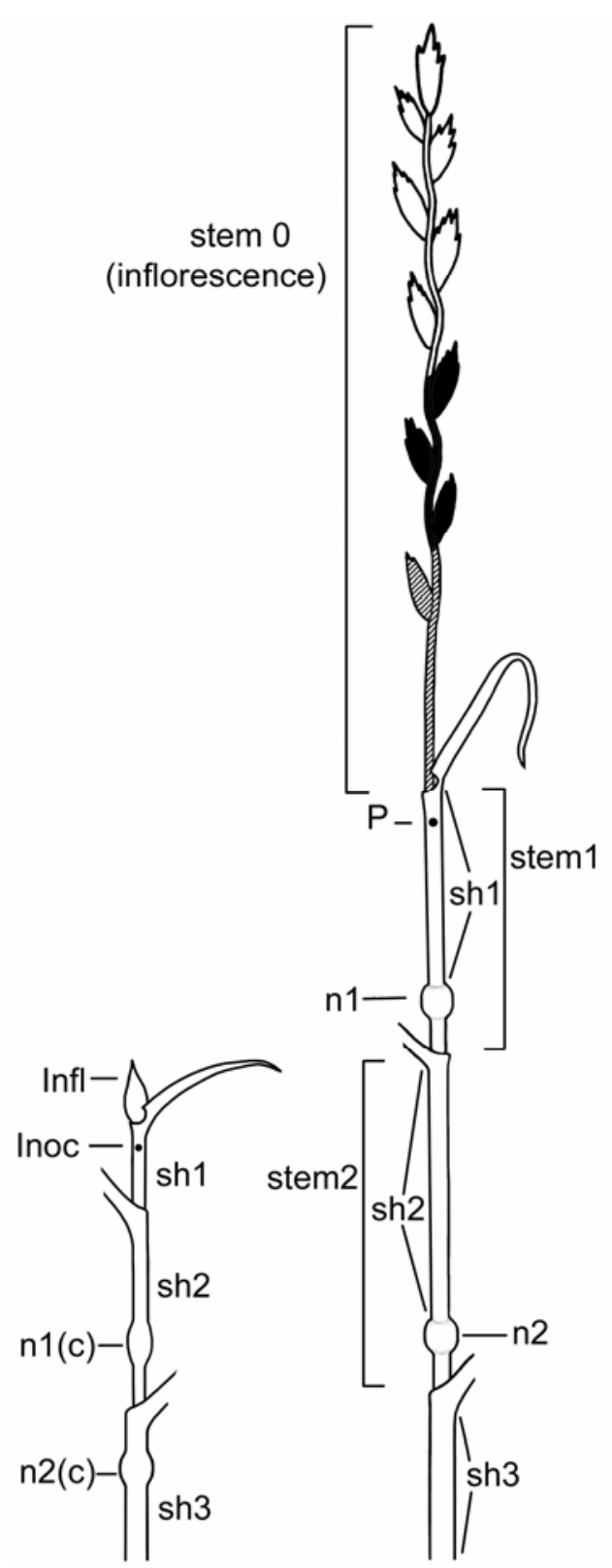

Fig. 1. Morphology of perennial ryegrass and the intraplant spread of stem rust. A developing grass tiller has a telescoped morphology, in which the upper (younger) segments emerge from within the enclosing leaf sheaths of older segments. For reference in this paper, we labeled the leaf sheaths from youngest $(\operatorname{sh} 1)$ to oldest $(\operatorname{sh} 3)$. Each sheath extends upward from its subtending node (n), e.g. sh1 extends from $\mathrm{n} 1$. The segment between the top of a sheath and the top of the next-lower sheath is designated a stem segment; the inflorescence (Infl) is designated stem0, and successively older stems are stem1 and stem2. In the diagram, Puccinia graminis is applied to an inoculation site (Inoc) on sh1 at Time 1, when $\mathrm{n} 1$ and $\mathrm{n} 2$ are covered (c) by $\operatorname{sh} 2$, much of sh1 is still enclosed in sh2, and Infl is just emerging from within sh1. By Time 2, the tiller has extended so that $n 1$ and $n 2$ are exposed, and stem0 (Infl) has elongated to its full length. At Time 2, intraplant spread of the pathogen has occurred as new infections have arisen on stem0, due to inoculum released from the sporulating lesion on the inner surface of the pustule $(\mathrm{P})$ on sh1. Infections on Infl that occurred $>1$ latent period before Time 2 have now erupted (indicated by the black fill), and infections that occurred $<1$ latent period before Time 2 have not yet erupted (indicated by hatched fill). The upper portion of Infl moved past the Inoc site during the first latent period after Time 1, when there was not yet any inoculum released from the pustule on sh1, and is therefore disease-free (indicated by no fill). 
initially becomes visible, were set to zero (i.e., there are no negative lengths for visible segments).

For the $\mathrm{x}$-axis quantity, we used thermal time (heat units) instead of chronological time because the model for secondary disease spread will be a component of an epidemic model that runs on thermal time of the pathogen; specifically the latent period duration (5). The time unit (latent period unit [LPU]) is equal to the thermal time required for one latent period of the stem rust pathogen on perennial ryegrass; $1 \mathrm{LPU}$ is approximately equivalent to 146 degree-days calculated with lower and upper thresholds of 1 and $28^{\circ} \mathrm{C}$, respectively (5). LPU per 15 min was calculated from the 15-min air temperature $(T)$ data, using the previously published (5) formula:

$\mathrm{LPU} / \mathrm{h}=0.5\{(0.000156 T-0.000206)(1-\exp [0.497(T-35.5)])\}$

For temperatures $<1.4^{\circ} \mathrm{C}$ or $>35.5^{\circ} \mathrm{C}, \mathrm{LPU}=0$. Cumulative values of LPU were calculated during the season with a reference value of 0 for the date when the inflorescence (stem 0 ) first became visible above the flag leaf sheath. Therefore, dates earlier than that reference time have negative values of LPU.

To fit equation 2 to the data for each plant segment, we used the nonlinear regression procedure in SigmaPlot software (SPSS, Inc., Chicago). The "4-parameter sigmoid", which is the same as equation 2 , was selected and a constraint was applied, with $y_{0}$ set to the desired truncation value $[d(a)]$ as described earlier in this section. The fitted equation and its associated parameters were accepted if the adjusted regression coefficient was at least 0.90 .

Intraplant disease spread. Three field experiments, independent of the experiments for tiller elongation, were conducted to quantify spread of stem rust from the flag sheath to the inflorescence in individual, infected tillers. In the first experiment, carried out in 2005, measurements were made and fungicide treatments were applied to tillers with partially extended inflorescences and with multiple sporulating lesions on the flag sheath. In two experiments conducted in 2006, tillers were individually inoculated at a single site on the flag sheath when the inflorescence was newly emerging, and fungicide treatments were applied at one of two later dates. In all three experiments, observed development of disease on the inflorescence was compared with model predictions.

2005 Experiment. Seed of perennial ryegrass cv. Morningstar was planted in the field in September 2004. In March 2005, individual plants were dug, potted, and taken to the greenhouse for inoculation with urediniospores of $P$. graminis subsp. graminicola. Inoculation procedures were as previously described (5), using a spore suspension of $0.6 \mathrm{mg} / \mathrm{ml}$ of Soltrol oil. These plants were at the rosette stage, with no tiller extension evident. After incubating plants overnight under conditions favorable for infection (5), they were transplanted into field plots of perennial ryegrass cv. Manhattan 4, which displays a moderate level of resistance to stem rust (7). The plots of Manhattan 4 were 3 by $1.2 \mathrm{~m}$ in size, and one inoculated Morningstar plant was replanted into each plot. On 3 June 2005, individual Morningstar tillers with rust symptoms on the flag sheath were labeled, and measurements were made of flag sheath length, inflorescence length, and extent and locations of rust pustules on the sheath. A fungicide application at standard labeled rate was then made, in a completely randomized design, to each 3-by-1.2-m block containing a labeled tiller. Fungicide treatments were propiconazole (Tilt; propiconazole at $189 \mathrm{~g}$ a.i./ha), azoxystrobin (Quadris; azoxystrobin at $165 \mathrm{~g}$. a.i./ha ), or nontreated. Tilt and Quadris are products of Syngenta Corp. (Basel, Switzerland). The azoxystrobin solution also included a nonionic surfactant at $1 \% \mathrm{vol} / \mathrm{vol}$ (Agridex, Helena, MT). Fungicides were applied with a $\mathrm{CO}_{2}$ backpack sprayer with XR Teejet 8003VS nozzles (R\&D Sprayers, Opelousas, LA) in 187 liters/ha at a pressure of $138 \mathrm{kPa}$. Additional tillers in which no disease had developed on the flag sheath were designated as nonsprayed, nondiseased checks. There were eight replicates per treatment and one tiller per replicate. At 10 and 21 days later, sheath and inflorescence length was recorded for each tiller and the location and extent of all erumpent stem rust pustules on the flag sheath and inflorescence were recorded. Severity of stem rust on the inflorescence was quantified as the sum of the length of all lesioned portions of the inflorescence (e.g., a severity of $8 \mathrm{~cm}$ would be assigned to an inflorescence that had individual lesions occupying $0.5-, 3.0-, 2.5-$, and $2.0-\mathrm{cm}$ lengths of the inflorescence). Air temperature at canopy height was recorded at 15 -min intervals throughout the study by means of a thermistor probe housed in a ventilated shield and attached to a data logger (Campbell Scientific Inc.).

2006 Experiments. Two field experiments were conducted in separate plantings of perennial ryegrass, $\approx 0.5 \mathrm{~km}$ from each other. Both stands were planted from seed (cv. Morningstar) in October 2005 and maintained according to standard agronomic practices, including adequate nitrogen fertilization. The two experiments were initiated (inoculated) on different days in May but the experimental design in the two experiments was the same. In late May 2006, treatment plots, each 1.2 by $1 \mathrm{~m}$, were designated in the plantings and fungicide treatments were assigned in a completely randomized design, with five replicate plots per treatment. Within each replicate plot on the first day of each experiment, five tillers were identified that had the inflorescence tip located between $0.5 \mathrm{~cm}$ below and $0.5 \mathrm{~cm}$ above the flag leaf ligule. All selected tillers, as well as all other plants in the experimental stands, were free of disease on the starting date. Each tiller was individually tagged to maintain data identity throughout the experiment and the length of the flag sheath was recorded. Each tagged tiller was then inoculated at a single location on the flag sheath. Inoculum was a suspension of urediniospores in Soltrol oil $(4 \mathrm{mg} / \mathrm{ml})$; spores had been collected from a field planting in June 2005 , then desiccated, stored at $-60^{\circ} \mathrm{C}$, and heat shocked immediately before use, as described previously (5). The inoculation site on the flag sheath, $\approx 2 \mathrm{~cm}$ below the ligule and on the opposite side from the seam produced by the overlapping edges of the sheath leaf, was rubbed gently between thumb and forefinger to reduce epidermal wax. Then, a size-00 paintbrush was used to apply an amount of spore suspension small enough to avoid any excess running down the sheath. Automated weather-monitoring equipment at each field site recorded overnight air temperatures and leaf wetness to verify infection favorability (6). Air temperature was monitored continually (15-min intervals) throughout the duration of each experiment for calculation of heat units (LPU) (5).

When $\approx 1.2$ latent periods had elapsed since the 2006 inoculation date, each tiller was examined to obtain measurements of sheath length, inflorescence length, and the presence and location of erumpent lesion on the sheath at the inoculation point. When $\approx 1.6$ latent periods had elapsed since inoculation (14 days in experiment 1 and 13 days in experiment 2), "early-spray" fungicide treatments were applied. Propiconazole or azoxystrobin was applied using the methods described for the 2005 experiment. After an additional 5 days had elapsed, "late-spray" fungicide treatments were applied to other plots. The check (nonspray) plots received no fungicide. Immediately before fungicide application and at several subsequent times up to 1 month after inoculation, each tiller was measured to obtain the lengths of the sheath and inflorescence. The locations and extent of any erumpent stem rust pustules on the inflorescence (stem 0 ) were recorded also.

Severity of the disease on the inflorescence was calculated as described for the 2005 experiment. In some cases, one or more of the five inoculated tillers per plot did not become infected at the sheath inoculation site but there were at least three infected tillers per plot in every case. For data analysis, the values from all successfully infected tillers within a replicate plot were averaged to produce the replicate value for plant segment length and for 
disease severity. There was no disease on the inflorescence of any tiller which lacked a lesion on the sheath (noninoculated checks or tillers with failed sheath inoculations).

Construction of model for infection extent. A model was created to combine information about plant growth with infection dynamics and fungicide effects. The independent variable in the model is thermal time (in LPU) calculated from air temperature measurements (5). Modeled processes were as follows. Time of infection is considered to be $2 \mathrm{~h}$ after sunrise on the morning following inoculation (6). Beginning with this initiation of an infection event on the flag sheath surface, a pustule becomes erumpent 1.0 LPU later (as determined from air temperature measurements). At the time (i.e., day and hour) it becomes erumpent, this source pustule begins to deposit spores from its inner surface onto the inflorescence that is emerging from within the sheath (8). From that time forward, each unit length of inflorescence elongation results in exposure of that unit length of inflorescence to inoculum from the source pustule, and thus to intraplant infection by the fungus. The model for this process, fundamental to the overall modeling of disease severity in the presence or absence of fungicide, was implemented as a simulation. The simulation time step was $0.1 \mathrm{LPU}$, beginning with the eruption of the source infection at the inoculation site on the sheath. For each time step, the increment of inflorescence length exposed to inoculum was calculated as the increment of growth determined from equation 2 parameterized (Table 1) for the inflorescence. Previous research (8) showed that the average probability of infection by this process was 0.9 (i.e., for each $1 \mathrm{~cm}$ length of stem exposed to inoculum [modeled stem growth after eruption of the source pustule], $0.9 \mathrm{~cm}$ of lesions actually occur on average). Therefore, the calculated length increment for a 0.1 -LPU time step was multiplied by 0.9 to model average centimeters of infection in a population of inflorescences subject to this process. In the simulation, all infections within a 0.1 -LPU cohort are assigned the same age. Therefore, a cohort of infections which occupies a length equal to 0.9 times the inflorescence growth increment occurring within that time step are modeled to erupt (become visible) 10 time steps $(10 \times 0.1 \mathrm{LPU})$ after their initiation. By this time, elongation of the inflorescence may have carried infection sites some distance above the top of the flag sheath (Fig. 1). The location of erumpent pustules on the inflorescence at any given observation date (and its associated cumulative LPU) depends on thermal time acting through the fungus latent period and plant elongation.

Construction of model for fungicide effects. To model the effects of fungicide on the process, inflorescence infection sites are classified into one of two categories: (i) sites that already have been carried above the top of the sheath when fungicide is applied and, therefore, are exposed to direct fungicide contact; and (ii) sites still covered by the sheath when fungicide is applied. The latter includes sites where infection has already occurred but which are still covered by the sheath and sites that are not yet in a position for infection (are below the level of the sheath lesion) at the time of fungicide application but will be in such a position as inflorescence elongation continues after that time. For type I sites (infected sites receiving direct fungicide contact), previously published equations (9) calculate fungicide effect based on the age of the infection (in LPU) at the time of fungicide exposure, with younger infection sites (e.g., those infected 1 day before fungicide application) having a higher mortality rate than older infection sites (e.g., those infected a week before application). These equations (9) are

for propiconazole: proportion killed $=0.968-0.0634 x-0.49 x^{2}-0.101 x^{3}$

for azoxystrobin: proportion killed $=0.953-0.0946 x-0.111 x^{2}$

The $x$ value is time expressed as LPU between fungicide application and the start of an infection; negative values of $x$ indicate that application occurred before infection. Proportion killed is constrained to be 0 to 1.0 , inclusive. This constraint means that the equations have a domain (LPU values) that runs from $\approx 2$ LUP pre-infection to 1 LUP postinfection, reflecting the fact that the fungicides have a residual (postapplication) effect that persists no more than 2 LUP and are ineffective in postinfection situations if the pustule has already erupted (i.e., after 1 LUP postinfection). As previously indicated, the simulation model produces cohorts of secondary infections in 0.1-LUP time steps. Therefore, each cohort has a defined age at the time of fungicide application and this age (or the time since fungicide application in the case of postfungicide infection) is used to apply the fungicide effects (equations 4 and 5). Thus, in the postinfection fungicide case, different cohorts will be differently affected by fungicide application because their ages differ. In the case of postfungicide infection, different cohorts will be affected differently as the fungicide effect attenuates with time.

In type II sites (which are not yet pushed out from the enclosing flag sheath when fungicide is applied), mortality of current and future infections depends on the fungicide and is modeled to be independent of time. A previous study (9) indicated that application of propiconazole reduces the probability of viability from 0.9 (the base condition, as described in the previous paragraph) to 0.7 , whereas azoxystrobin reduces the probability to 0.07 . A pustule that survives the effect of fungicide is modeled to erupt (become visible as disease) on the same schedule as any pustule formed in the absence of fungicide (i.e., 1.0 LPU after infection begins). Viability probabilities for this class of infection site are multiplied by inflorescence exposure length (calculated from equation 2) to produce an expected value for average severity, expressed as length of stem (in centimeters) with lesions. Some pustules covered by the sheath at the time of fungicide application are already erumpent (i.e., initiated $\geq 1.0$ LPU before fungicide application) and they are modeled to be unaffected by

TABLE 1. Parameters for segment length equation derived from field observations of perennial ryegrass ${ }^{\mathrm{y}}$

\begin{tabular}{|c|c|c|c|c|c|}
\hline \multirow[b]{2}{*}{ Plant segment } & \multicolumn{4}{|c|}{ Parameter $^{\mathrm{Z}}$} & \multirow[b]{2}{*}{ Adjusted $r^{2}$} \\
\hline & $a$ & $b$ & $c$ & $d$ & \\
\hline Inflorescence & 37.5 & 1.245 & 0.718 & 0.13 & 0.98 \\
\hline Stem 1 & 21.6 & 0.201 & 0.460 & 0.18 & 0.95 \\
\hline Stem 2 & 18.3 & -0.755 & 0.604 & 0.32 & 0.93 \\
\hline Stem 3 & 13.6 & -1.764 & 0.546 & 0.34 & 0.90 \\
\hline Sheath 1 (flag sheath) & 17.3 & -0.033 & 0.378 & 0.22 & 0.96 \\
\hline
\end{tabular}

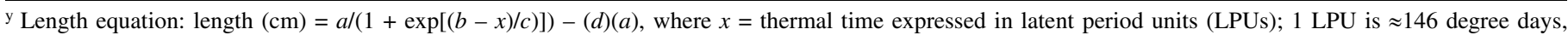
calculated with a lower threshold of $1^{\circ} \mathrm{C}$. LPU is arbitrarily set to 0 for the date of inflorescence emergence, and has negative values for earlier dates. Length data

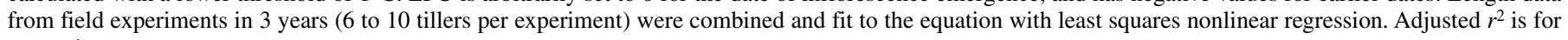
regression.

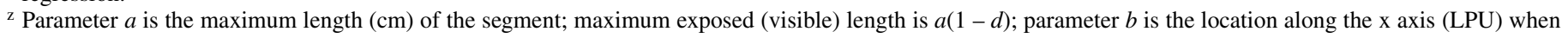
the segment is at one-half its maximum length; and parameter $c$ represents the steepness (dimensionless) of the curve. 
the fungicide. On any given day, the modeled lengths of erupted disease (older than 1 LPU, therefore visible as pustules) from the two classes of infection sites are summed for expected severity. These expected values were compared with the averages of observed severity values (expressed as visibly diseased length of inflorescence) derived from field experiments.

\section{RESULTS}

Plant growth modeling. The sigmoid equation (equation 2) was readily fit, by means of nonlinear regression in SigmaPlot (SPSS, Inc.), to lengths of inflorescence, flag sheath, and stems 1 to 4 recorded in the data sets of observations from 2004, 2005, and 2007. Parameters for the fitted equations are listed in Table 1. The modeled visible final length $[a(1-d)]$ of the inflorescence, flag sheath, and stems 1,2 , and 3 were $32.6,13.5,17.7,12.4$, and $9.0 \mathrm{~cm}$, respectively. These curves (Fig. 2) gave a good fit to the observed data, with $r^{2}$ values of 0.90 to 0.98 for the different segments (Table 1).

The fitted curves were used in the model for intraplant spread of rust and the effects of fungicide on that spread. In particular, we used the growth model of the inflorescence and of the flag leaf sheath (the segment which initially encloses the inflorescence) in the simulation model. For the model assessment described in the following sections, the parameters fitted to the plant-growth data from 2004, 2005, in 2007 were used to run simulations for data sets collected in independent experiments conducted in 2006 and 2007. The 2007 disease and plant-length experimental data were collected in a different experiment at a different location (different experimental field) from the 2007 plant-growth data used for parameterizing the plant growth equations. As previously described, the simulation model has a time step of 0.1 LPU; therefore, the change in length for each time step was calculated as length at time $t$ minus the length at time $t$ minus 0.1 LPU.

Modeling of disease and fungicide effects: 2005 experiment. On 3 June, the date fungicide treatments were applied, there were erumpent pustules on the flag sheaths that could function as

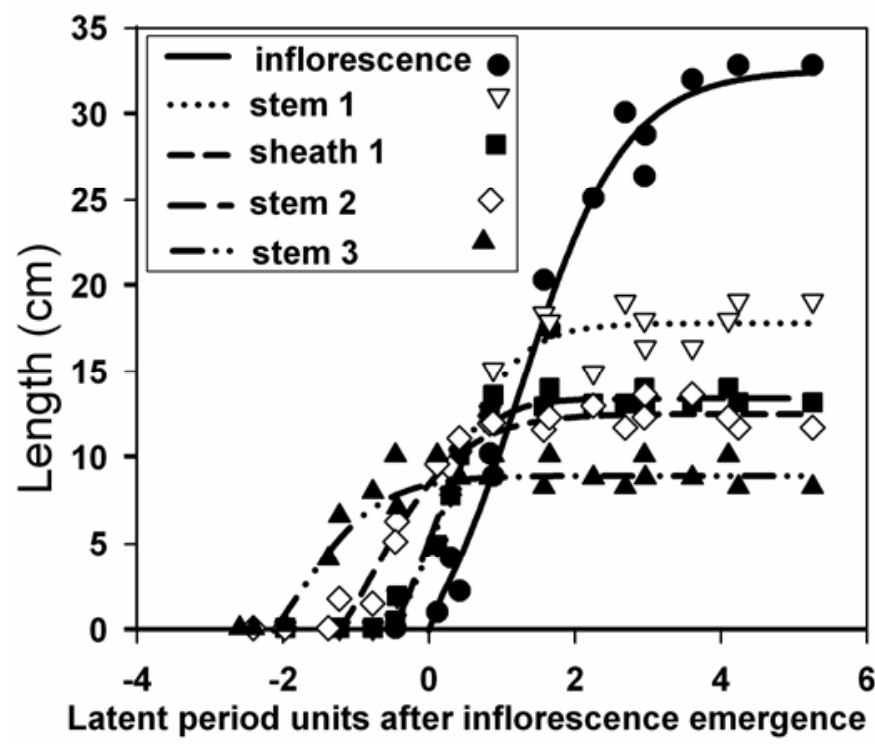

Fig. 2. Elongation of segments in perennial ryegrass. Lengths of stem and sheath segments of perennial ryegrass cultivar Morningstar during tiller extension. Time is expressed in thermal units, where 1 latent period unit (LPU) is the duration of the stem rust latent period on perennial ryegrass, approximately 146 degree days. LPU is arbitrarily set to 0 for the time when the inflorescence first emerges from the flag sheath. Data (symbols) are from field observations in three different years, and each symbol represents the average of at least six tillers. Equations (lines) were fit to the data by least squares nonlinear regression (Table 1). source pustules for intraplant spread of the disease onto the emerging inflorescences. These source pustules averaged $3.4 \mathrm{~cm}$ in length, with a range of 0.5 to $7.9 \mathrm{~cm}$, and the average location of the uppermost sheath lesion was $0.8 \mathrm{~cm}$ below the flag sheath ligule, with a range of 0.2 to $2.6 \mathrm{~cm}$. In applying the rust model to this plant material, certain assumptions were required because the initiation time of the observed, erumpent sheath lesions was not precisely known. These sheath lesions were assumed to result from infections that originated on the first day that the infection site on the sheath had emerged and, thus, was available for infection by airborne spores. For example, a pustule located $2.5 \mathrm{~cm}$ from the top of the sheath was assumed to result from an infection that occurred on the day that the visible sheath length first exceeded $2.5 \mathrm{~cm}$, as estimated by the plant-growth model. To address difficulties with modeling the overlapping of infections on the inflorescence that could result from sheath lesions at multiple locations, we made the assumption that the uppermost pustule on a sheath (earliest to occur) was the lesion that acted as the source for the secondary infections on the inflorescence.

Observed values of disease severity expressed as summed length of lesions on the inflorescence, and the associated variance expressed as standard deviation, are presented in Figure 3. On the final postfungicide observation date (24 June), disease severity (total lesion length) on the inflorescences averaged 17.6, 7.4, and $4.2 \mathrm{~cm}$ on the check, propiconazole-treated, and azoxystrobintreated tillers, respectively. The respective modeled values were 21.6, 12.7, and 5.4. The modeled values fell within \pm 1 standard deviation of the observed values (Fig. 3). We note that the variability of the observed material was quite large so that, in most cases, the model did not have to be extremely accurate to fit within this range of variation. The model outputs did, however, produce the same order of severities as in the observed data (e.g.,

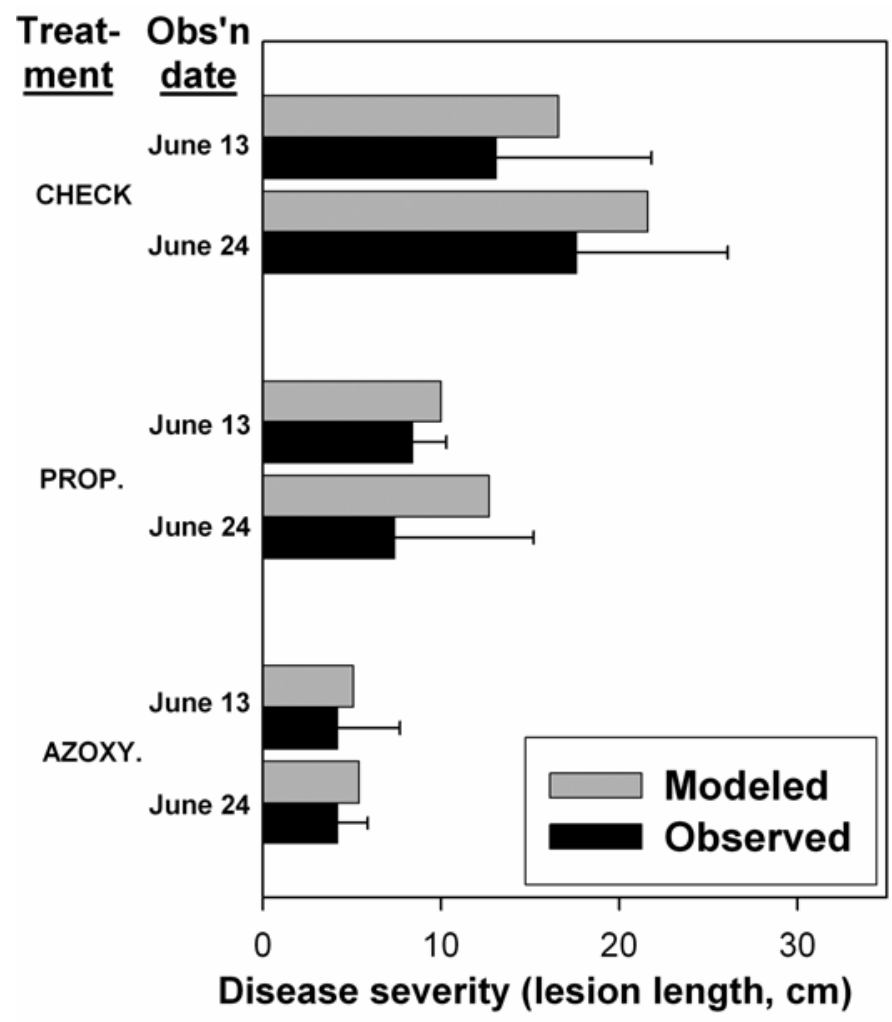

Fig. 3. Modeled and observed stem rust severity on inflorescences of perennial ryegrass in 2005 field experiment. Disease severity is the extent (cm) of disease along the length of inflorescence. Individual tillers were treated (check, propiconazole or azoxystrobin) on 3 June, when sporulating lesions were already present on the flag sheath, but not on the inflorescence. Error bars are standard deviation of observed disease lengths on the indicated observation dates. 
24 June observation of check treatment having the highest severity, 13 June observation of azoxystrobin-treated having the lowest severity), except for reversing the order of the 13 June check and 24 June propiconazole treatment model values. Also, the modeled and observed results agreed with respect to the rank order of severities among the fungicide treatments within each date. The probability of infection for a segment exposed to intraplant inoculation, which we had previously measured to be 0.9 (9), was found to be 0.8 in this 2005 experiment. That is, for every $1 \mathrm{~cm}$ of inflorescence length in the check tillers that was subject to possible intraplant infection, an average of $0.8 \mathrm{~cm}$ of lesion length occurred.

Modeling of disease and fungicide effects: 2006 experiments. For the two experiments conducted in 2006, the sheath infections were controlled so that model performance could be assessed more precisely. Ambiguity due to possible overlapping secondary infections from multiple-source pustules on the sheath was avoided, and the age and location of the source infections were known. In addition, observations were made at four times after secondary infections started, so that data from a more complete time course were available for assessment. Also, timing effects of the fungicide applications were tested by including two differently timed treatments for each fungicide.

The time at which the inflorescence first appeared above the flag sheath ligule was known (it was the day of inoculation) in these 2006 experiments, and this information was used to set the initiation time for the inflorescence extension model in each experiment; that is, the $b$ parameter in the extension equation (eq. 2) for the inflorescence was set so that the modeled inflorescence length was zero on the inoculation date. Observed inflorescence lengths in the first experiment on 28 May, 5 June, and 16 June were 17.3, 28.1, and $31.0 \mathrm{~cm}$ and modeled lengths for these dates were $16.1,25.3$, and $30.6 \mathrm{~cm}$, respectively. In the second experiment, observed lengths on 28 May, 5 June, and 16 June were $15.8,28.3$, and $33.5 \mathrm{~cm}$ and modeled lengths for these dates in experiment 2 were $14.3,24.4$, and $30.3 \mathrm{~cm}$, respectively.

In the first 2006 experiment, observed final disease severities (summed lesion length) on inflorescences averaged 0 to 0.6 for the early- and late-treated azoxystrobin applications, respectively, 4.8 and 5.0, respectively, for the propiconazole-treated tillers, and 13.4 in the nontreated tillers (Fig. 4). Observed severity decreased between the first and last observation date in the late-sprayed azoxystrobin treatment, because pustules that were counted on the first observation dates but that died soon after due to fungicide action and were partially obscured by regrowth of host tissue at the infection site were disregarded in measurements of "observed disease" at the later dates. The observed severities in the inoculated, nonsprayed check treatment across all observation dates were within $2.0 \mathrm{~cm}$ of the modeled lengths (Fig. 4). The model was within $1.5 \mathrm{~cm}$ of observed for all dates in the azoxystrobinsprayed treatments (early or late sprayed) and for the late-sprayed propiconazole treatments. In the early-sprayed propiconazole treatment, the difference between modeled and observed was $<0.5$ $\mathrm{cm}$ for the first two observation dates but 2.1 and 2.8 on the last two dates. The model accurately predicted the ranking of final disease severities among fungicide treatments (i.e., greatest severity in the nontreated tillers and progressively less in propiconazole-treated and azoxystrobin-treated tillers). Although both azoxystrobin treatments were correctly modeled to have $<1.0 \mathrm{~cm}$ of disease (less disease than the other treatments), the order between early- and late-treated was incorrectly modeled.

Modeling of the second 2006 experiment provided results generally similar to the first one, although final disease severity was slightly greater in this experiment for the check and early-sprayed propiconazole treatments. Modeled disease lengths were within $1.5 \mathrm{~cm}$ of observed lengths for 18 of 20 results (date-treatment); the model overpredicted the early-treated propiconazole and nontreated check by 1.7 and $1.9 \mathrm{~cm}$, respectively (Fig. 5). As in the first 2006 experiment, the ranking of final disease severities among treatments was correctly modeled, except for the relative ranking of the small, nearly identical severities in the azoxystrobin early- and late-sprayed treatments.

Combined data from all experiments. Overall accuracy of modeled disease is reflected in regression analysis of modeled versus observed disease lengths for all experiments (Fig. 6). The correlation coefficient (0.93) shows that, over the range of severities and conditions, the original model (designated version 1) adequately described the observed results. The slope of the observed versus modeled data is 1.1 and the intercept is $0.3 \mathrm{~cm}$. We also evaluated two modifications to the model. For the first modification (model version 2), we adjusted the ratio of the diseased/ exposed stem length. As noted earlier, previous observations (9) under greenhouse conditions suggested that the probability of successful secondary infection for any given site exposed to inoculum by the intraplant disease spread mechanism is 0.9 . In the field experiments described in this article, the measured value (in check treatments, where fungicide effects would not interfere with the phenomenon) was 0.7 to 0.9 across the experiments, with an average value of 0.8 . When the model was run using 0.8 instead of 0.9 as the infection probability for intraplant spread, the slope of the line for modeled versus observed was 0.98 , an improvement over the accuracy of the model when 0.9 was used as the infection probability (Fig. 6). We assessed a second modification (model version 3 ) to improve modeled effects of fungicides

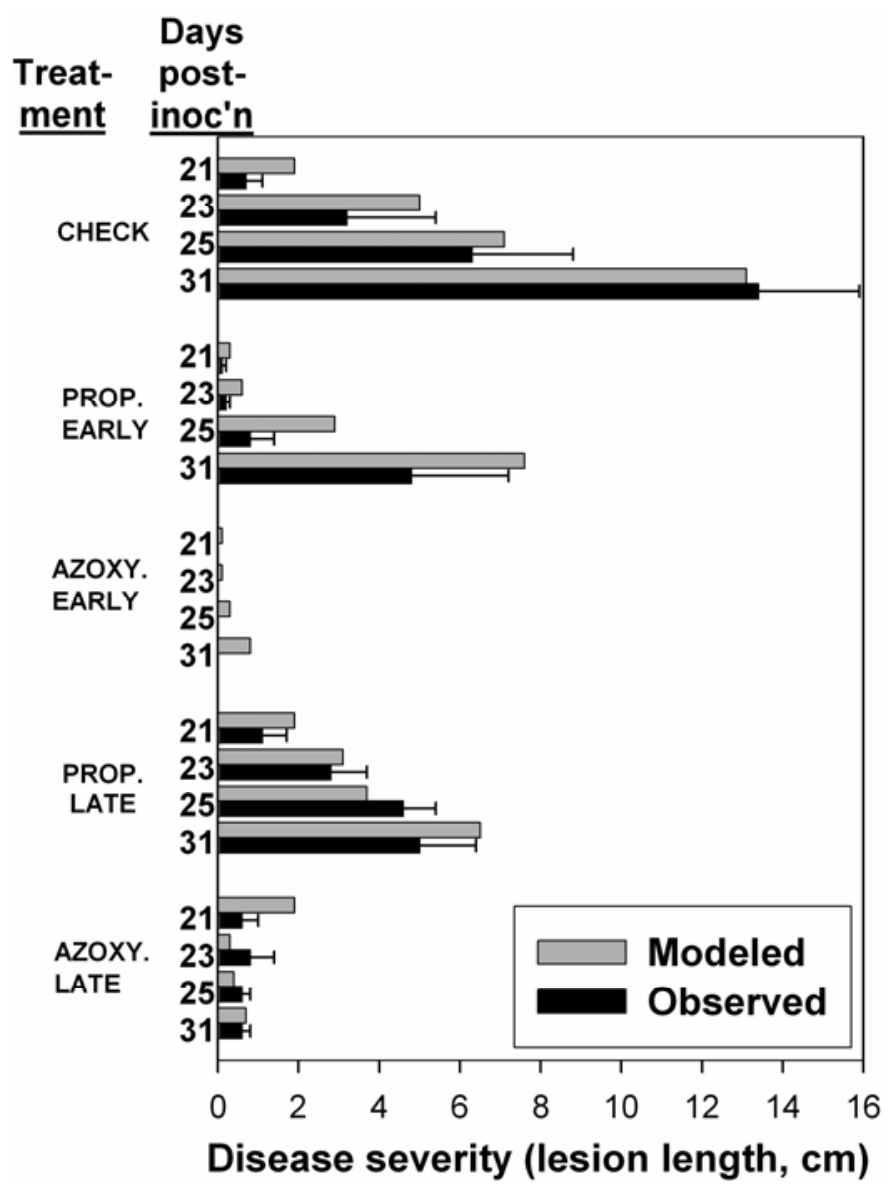

Fig. 4. Modeled and observed stem rust severity on inflorescences of perennial ryegrass in first of two 2006 field experiments. Disease severity is the extent $(\mathrm{cm})$ of disease along the length of inflorescence. Individual tillers were inoculated on the flag sheath when the inflorescence was just emerging. Fungicide (propiconazole or azoxystrobin) was applied at one of two later dates, and observations were made on four dates after fungicide application. Error bars are standard deviations of the observed values, five replicates per mean. Fungicides were applied 15 or 21 days after inoculating the flag sheath, for early or late applications, respectively. Disease extent measured at 21, 23, 25 , and 32 days after inoculating flag sheath. 
on type II infections (i.e., those covered by the sheath at the time of fungicide application). We noted that the previously derived (9) fungicide efficacy values (expressed as infection viability values of 0.7 and 0.07 for propiconazole and azoxystrobin, respectively) produced a bias toward overestimation of fungus survival. Revised fungicide efficacy values of 0.55 and 0.02 for survival when exposed to propiconazole and azoxystrobin, respectively, improved overall model performance. When applied to the modeledversus-observed data, these two modifications resulted in a correlation coefficient of 0.95 and a slope of 0.97 (Fig. 6).

\section{DISCUSSION}

The model for intraplant disease spread produced estimates of diseased inflorescence length that were within $2 \mathrm{~cm}$ of actual average diseased length for most observations in this three-experiment study, and were well correlated with them $\left(r^{2}=0.93\right)$. The truncated, simple sigmoidal equations for length of the plant segments, which formed the basis for modeling disease development (8), were adequate to describe our observations of perennial ryegrass growth. By incorporating previous results on efficacy of fungicides and their physical modes of action in relation to plant phenology (9), we were able to model severity of disease development as affected by fungicide application. The model performed

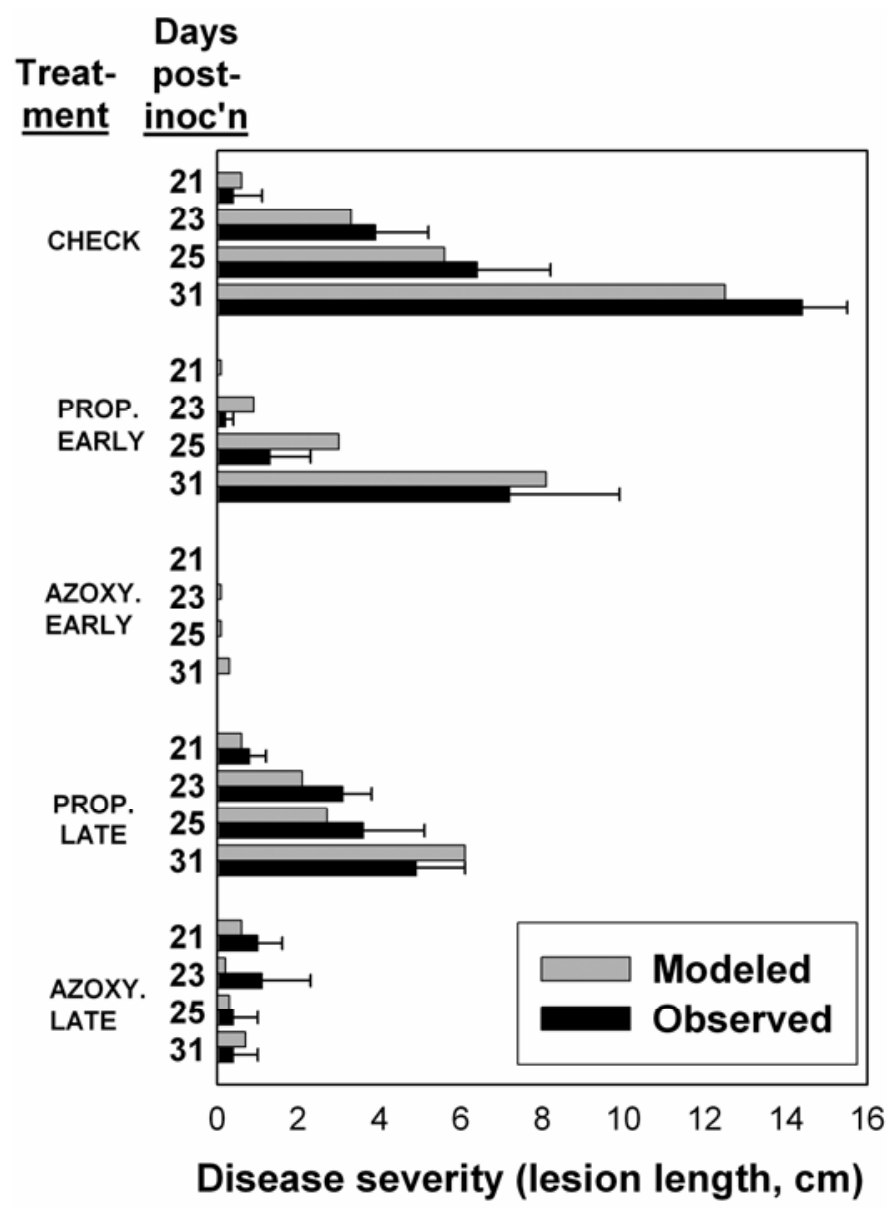

Fig. 5. Modeled and observed stem rust severity on inflorescences of perennial ryegrass in second of two 2006 field experiments. Disease severity is the extent $(\mathrm{cm})$ of disease along the length of inflorescence. Individual tillers were inoculated on the flag sheath when the inflorescence was just emerging. Fungicide (propiconazole or azoxystrobin) was applied at one of two later dates, and observations were made on four dates after fungicide application. Error bars are standard deviations of the observed values, five replicates per mean. Fungicides were applied 14 or 20 days after inoculating the flag sheath, for early or late applications, respectively. Disease extent measured at 20,22, 24, and 31 days after inoculating flag sheath. well (produced an accurate ranking of disease across a four-way factorial of fungicide type and timing) in the 2006 experiments, where the process of secondary infection from a single sheath lesion could be quantified, and also in the 2005 experiment, where multiple sheath lesions added more complexity to the observed process and more uncertainty to its measurement.

The disease model was somewhat biased, tending to overestimate disease severity. This was likely due to an overestimate of the probability of successful infection when an emerging segment is in contact with a sporulating lesion on the overlying sheath. When this ratio of diseased/exposed length was modified from 0.9 (the previously observed value in greenhouse experiments) (8) to 0.8 (as observed in these field experiments), the overestimation bias was largely removed. Further adjustment, by increasing fungicide efficacy values, reduced error variance in the regression analysis of observed versus modeled (Fig. 6). Adjustment in fungicide efficacy was particularly important to reduce the overestimation of disease in plants treated with propiconazole. With these adjustments, the slope of the regression line for modeled versus observed values was close to $1(0.97)$ and the correlation coefficient was 0.95 . More testing will be required to determine whether these modifications are generally applicable. However, even without these modifications, the original model adequately conveys expected disease severity under different management treatments.

As with any deterministic model, there are inaccuracies in representing the stochastically variable reality. One source of uncer-

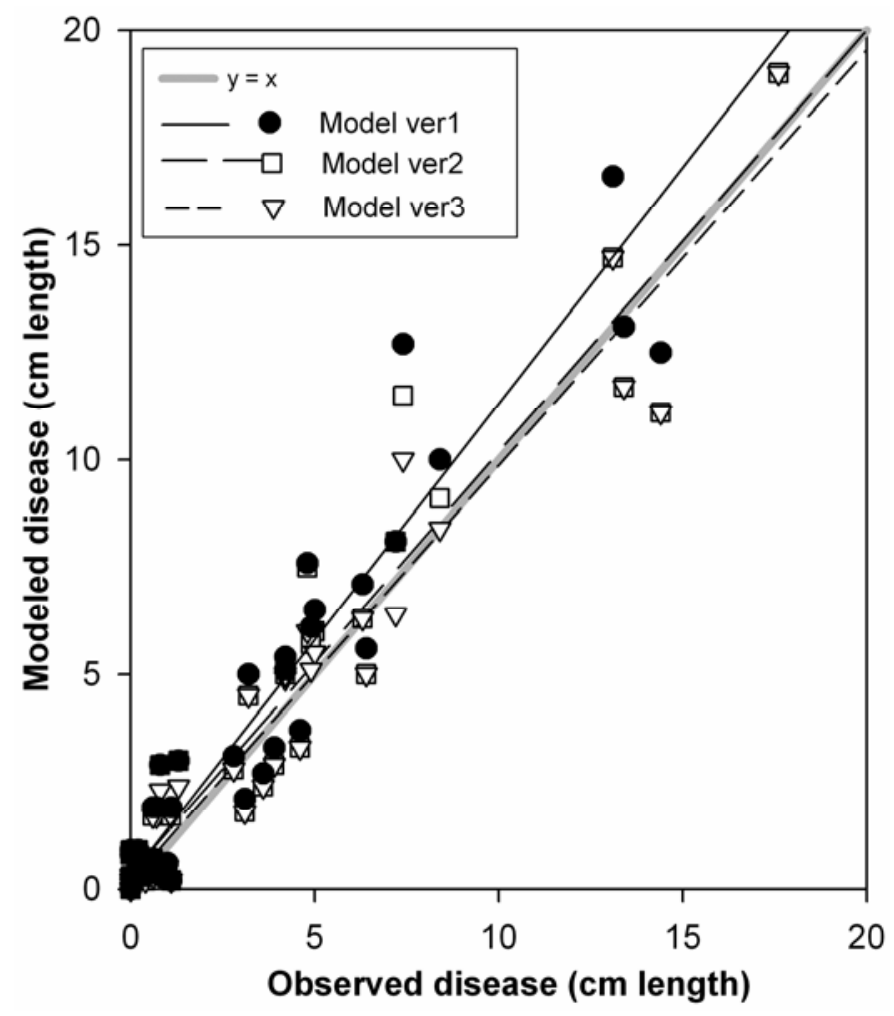

Fig. 6. Comparison of modeled versus observed stem rust severities. Each data point plots modeled and average observed disease severities for one observation date in one of the treatments (fungicide and timing, including check) in one of 3 field experiments conducted in 2005 and 2006. Three versions of the model are represented. The original version is as described in the Methods section. In version 2 of the model, the ratio of diseased to exposed inflorescence length is adjusted from its original value (0.9) to the value measured in the check treatments of the experiments $(0.8)$. In version 3 of the model, fungicide parameters are adjusted to reflect greater fungicide efficacy (described in text). Regression equations and coefficients are as follows. Original version: modeled $=1.1$ (observed $)+0.29$, adj. $r^{2}=0.93$; version 2 : modeled $=$ 0.98 (observed) $+0.34, r^{2}=0.92$; and version 3 : modeled $=0.97($ observed $)+$ $0.17, r^{2}=0.95$. 
tainty and inaccuracy in the model is its treatment of latent period duration. The model assumes that all infections have the same latent period whereas, in reality, there is a frequency distribution of latent period durations (5). The model uses the value of the duration required for $20 \%$ of the infections to become erumpent (5). Variability in latent period can have significant effects on the outcome of intraplant disease spread by affecting the timing of the secondary infections and their subsequent susceptibility to fungicide activity, which varies with age of the infection. Similarly, the model posits an "average" tiller to represent the growth dynamics of all tillers when, in fact, there is variability in length and growth rates (Fig. 2). Final length of the segments, particularly of the inflorescence, was variable among years, and this variability is not captured in the simple model. This uncertainty regarding stem growth must be considered in evaluating the robustness of the disease model presented here, as well as the full-stem rust epidemic model which is under development. In the experiments reported here, the disease model did not appear to be overly sensitive to inaccuracies in modeling inflorescence length, and such inaccuracies would not be expected to affect the modeled ranking of the efficacy of different management approaches (timing and choice of fungicide). Thus, the combined real variability of several biological processes is the cause of the observed variability in disease severity (Figs. 3, 4, and 5; error bars). The model attempts to estimate the midpoint of the disease outcomes well enough to inform management decisions, and it appears to do so.

Additional improvements that remain to be made in the model include modifications to encompass differences in growth dynamics among cultivars. Research is in progress to characterize these differences. Also, we noted that a significant factor in the overprediction of disease for the azoxystrobin-treated plants is the lack of a model component for pustules that die prematurely after they are formed, due to fungicide exposure. Pustules that died soon after eruption, and that were then partially obscured by regrowth of host tissue at the infection site, were not included in measurements of observed disease. Such pustules do not contribute to further epidemic development and probably have little or no effect on crop yield. The model should be adjusted to account for this component.

Given the satisfactory performance of the model for estimating intraplant spread from the flag sheath to the inflorescence, and the geometric similarity among internodes during stem extension, it is reasonable to apply the same modeling approach to all internodes of the extending grass plant as it produces flowering tillers. Furthermore, it is probable that the model can be successfully adapted to stem rust in other crops such as wheat. Other authors have noted the important effect of fungicide timing in the efficacy of stem rust control in wheat (3), including significant differences in preventing stem rust development on the peduncle (stem) of the inflorescence. The relationships between plant growth, intraplant spread, and fungicide activity, as approximated by the model presented here, help explain the importance of fungicide application timing for stem rust in perennial ryegrass, and might apply to wheat stem rust as well. For example, propiconazole is relatively more effective against ryegrass stem rust if applied after the secondary infections on the elongating stem have emerged from the protection of the sheath but before the infection process has continued far into the latent period. In contrast, azoxystrobin is relatively more effective when applied early in the intraplant spread process, when it can stop initiation of the new secondary infections on the stem by halting sporulation from the sheath lesion. This demonstration supports the use of epidemiological models for clarifying the appropriate role and optimum use for fungicides in disease management $(1,11)$.

\section{LITERATURE CITED}

1. de Kraker, J., van den Ende, J. E., and Rossing, W. A. H. 2005. Control strategies with reduced fungicide input for Botrytis leaf light in lily - a simulation analysis. Crop Prot. 24:157-165.

2. Lawless, C. Semenov, M. A., and Jamieson, P. D. 2005. A wheat canopy model linking leaf area and phenology. Eur. J. Agron. 22:19-32.

3. Loughman, R., Jayasena, K., and Majewski, J. 2005. Yield loss and fungicide control of stem rust of wheat. Aust. J. Agric. Res. 56:91-96.

4. Milne, A. Paveley, N., Audsley, E., and Parsons, D. 2007. A model of the effect of fungicides on disease-induced yield loss, for use in wheat disease management decision support systems. Ann. Appl. Biol. 151:113125.

5. Pfender, W. F. 2001. A temperature-based model for latent period duration in stem rust of perennial ryegrass and tall fescue. Phytopathology 91:111116.

6. Pfender, W. F. 2003. Prediction of stem rust infection favorability, by means of degree-hour wetness duration, for perennial ryegrass seed crops. Phytopathology 93:467-477.

7. Pfender, W. F. 2004. Effect of autumn planting date and stand age on severity of stem rust in seed crops of perennial ryegrass. Plant Dis. 88:1017-1020.

8. Pfender, W. F. 2004. Role of phenology in host susceptibility and withinplant spread of stem rust during reproductive development of perennial ryegrass. Phytopathology 94:308-316.

9. Pfender, W. F. 2006. Interaction of fungicide physical modes of action and plant phenology in control of stem rust of perennial ryegrass grown for seed. Plant Dis. 90:1225-1232.

10. Royo, C., and Blanco, R. 1999. Growth analysis of five spring and five winter triticale genotypes. Agron. J. 91:305-311.

11. Schoeny, A., and Lucas, P. 1999. Modeling of take-all epidemics to evaluate the efficacy of a new seed-treatment fungicide on wheat. Phytopathology 89:954-961.

12. Welty, R. E., and Azevedo, M. D. 1994. Application of propiconazole in management of stem rust in perennial ryegrass grown for seed. Plant Dis. 78:236-240. 